\title{
The immunology of gluten sensitivity beyond the intestinal tract
}

\author{
Aristo Vojdani, Ph.D., M.T.1*; Thomas O'Bryan, D.C., C.C.N., D.A.C.B.N. ${ }^{2}$ \\ ${ }^{1}$ Immunosciences Lab., Inc., 8693 Wilshire BIvd., Ste. 200, \\ Beverly Hills, CA 90211 \\ ${ }^{2} 28379$ Davis Parkway, \#801, \\ Warrenville, IL 60555
}

* Corresponding author, address, and telephone number: 8693 Wilshire Blvd, Suite 200, Beverly Hills, CA 90211 Phone (310) 657-1077; Fax (310) 657-1053 E-mail: immunsci@ix.netcom.com

Gluten sensitivity, celiac disease (CD) and gluten-sensitive enteropathy are terms that have been used synonymously to refer to a disease process affecting the small bowel 
and characterized by gastrointestinal symptoms and malabsorption. However, since 1966 scientific evidence has been accumulated demonstrating that gluten sensitivity can exist even in the absence of enteropathy. For example, patients with dermatitis herpetiformis and presentation of blistering skin do not have any gastrointestinal symptoms but have elevated gliadin antibody in the blood which improves on a gluten-free diet. ${ }^{1}$ Additionally, associations of CD with the involvement of other organs such as the central and peripheral nervous systems also go as far back as $1966 .^{2}$ However, until recently, this phenomenon of immune reaction against neural tissue, in particular the cerebellum, was attributed to vitamin deficiencies and not to immunological pathogenesis. During the past five years, based on overwhelming evidence of immune pathogenesis involving organs other than gut and skin, many scientists have begun to re-evaluate the notion that gluten sensitivity is solely a disease of the gut. Other organs suspected of involvement include: the joint, ${ }^{3-11}$ the heart, ${ }^{12-15}$ thyroid, ${ }^{16-18}$ bone, ${ }^{19,}{ }^{20}$ the brain cerebellum and the neuronal synapsins ${ }^{20-40}$ which are summarized below. Although it is believed that the prevalence of $\mathrm{CD}$ is one in one hundred, for every symptomatic patient with CD there are eight patients with CD with no GI symptom. In addition $10 \%$ of the healthy population have significant elevation in gliadin antibody but no obvious classic disease manifestations. In our laboratory, when the blood of these individuals is tested against different tissue antigens (joint, myosin, endothelial cell, bone antigens, myelin basic protein, cerebellar and synapsin peptides) more than $90 \%$ of them exhibit elevation in $\operatorname{IgG}, \operatorname{IgM}$ and $\operatorname{IgA}$ antibodies against one or all these organ-specific antigens. Therefore, gluten sensitivity beyond the gut can induce multi-organ system disorders, some of which are described below.

\section{A. The gut-joint axis: cross-reactive food antibodies in rheumatoid arthritis}

Patients with rheumatoid arthritis (RA) often feel there is an association between food intake and rheumatoid disease severity. In a recent study of this putative immunological link between gut immunity and RA, food antibodies were measured in serum and perfusion fluid from the jejunum of RA patients and healthy controls to determine the systemic and mucosal immune response. IgG, IgA and IgM antibodies to dietary antigens were measured in serum and jejunal perfusion fluid from 14 RA patients and 20 healthy subjects. The antigens originated from cow's milk ( $\alpha$-lactalbumin, $\beta$ lactoglobulin, casein), cereals, hen's egg (ovalbumin), cod fish and pork meat. In the intestinal fluid of many RA patients, all three immunoglobulin classes showed increased food specific activities, including gliadin antibodies. ${ }^{3,4}$

It is well-known that some $80 \%$ of untreated RA patients have been shown to have reduced maximum gastric acid output leading to a marked reduction in dietary protein degradation, which contributes to enhanced food immunoreactivity. ${ }^{5-7}$

A germ-free state prevents the development of gut and joint inflammation in HLA-B27 transgenic rats, thereby giving strong support to a connection between mucosal immunity and arthritis. Also, reactive arthritis in humans appears to be caused by a combination of a mucosa-associated microbial impact and genetic predisposition. Interestingly, some $90 \%$ of patients with reactive arthritis or ankylosing spondylitis express HLA-B27, and these disorders can be associated with Crohn's disease, ulcerative colitis, and jejuno-ileal bypass surgery - again emphasizing the putative gut-joint axis 
which is also supported by shared homing properties of activated intestinal immune cells. ${ }^{8-11}$

Moreover, animal experiments have demonstrated a wide-spread tissue distribution of food antigens shortly after feeding, which could predispose to synovial immune complex formation and thereby autoimmune joint reactions. Additionally, it was reported that intestinal levels of IgM and IgA are increased in patients with ankylosing spondylitis related to disease activity. Antigens from the gut microbiota rather than food are apparently involved in that disease..$^{7-8}$

of Key concepts and clinical implications:

The production of cross-reactive antibodies is strikingly increased in the gut of many RA patients. Their food-related problems might reflect an adverse additive effect of multiple modest hypersensitivity reactions mediated, for instance, by immune complexes promoting autoimmune reactions in the joints.

- Patients with joint problems, in particular rheumatoid arthritis, should be examined for the presence of $\operatorname{IgG}$ and $\operatorname{IgA}$ antibodies against dietary proteins and peptides including gliadin and casein.

- These patients should also be tested for neuroimmunology of gluten sensitivity, celiac disease and the associated autoantibodies.

\section{A1. Gluten sensitivity and its association with autoimmune myocarditis}

Myocarditis can be associated with systemic autoimmune disorders that if unrecognized and untreated can prevent the recovery of or even worsen myocardial function.

Several studies have demonstrated a close association between $\mathrm{CD}$ and autoimmune disorders, such as insulin-dependent diabetes mellitus, thyroid disorders, Addison's disease, and connective tissue disorders. An increased prevalence of CD (5.7\%) has been recently recognized in patients with idiopathic dilated cardiomyopathy, and an immunologic associative mechanism has been suggested. ${ }^{12}$

Although a reciprocal negative interaction between the heart and small intestine is known to occur whenever either organ is severely compromised, less recognized is the possibility of simultaneous damage of the 2 organs due to a common pathogenetic mechanism. A recent study showed the presence of an intestinal inflammatory disease in a large population of patients with myocarditis, with a prevalence that was 14 times higher than that in normal control subjects. ${ }^{12,13}$

This amount appears to be rather reliable, because for both anti-endothelial cell antibodies (AEA) and tTG antibodies, a high sensitivity ( $95 \%$ and $100 \%$, respectively) and specificity ( $90 \%$ and $100 \%$, respectively) have been found. Moreover, every single patient with antitransglutaminase antibodies and symptoms of autoimmune myocarditis was found to be positive for anti-heart antibody. These patients' clinical manifestations of myocarditis and heart failure markedly improved after a gluten-free diet or a combination of gluten-free diet and immunosuppressive therapy. Prior to administration of the gluten- 
free diet, these patients with chronic heart failure failed to respond to supportive treatment administered for more than six months. ${ }^{14}$ This responsiveness to a gluten-free diet and immunosuppressive therapy strongly suggest the existence of an autoimmune disorder directed toward antigenic components of both the myocardium and small bowel. Indeed, both myocarditis and gluten sensitivity are known to occur in association with systemic autoimmune disorders. The observation that these entities can be combined in the same patient is of clinical relevance. ${ }^{15-17}$

In fact, gluten sensitivity is invariably associated with an increase of intestinal permeability, which could lead to the translocation of many intestinal luminal antigens (such as ingested food proteins, bacterial breakdown products, endotoxins, heat shock proteins and active enzymes) that can exacerbate myocardial inflammation. Indeed, some other extraintestinal findings of $\mathrm{CD}$, such as chronic unexplained hypertransaminasemia, are attributed to the mechanism of antigenic overload. ${ }^{12}$

Furthermore, active $\mathrm{CD}$ is accompanied by consistent production of $\operatorname{IgA}$ autoantibodies to reticulin, a common constituent of the extracellular matrix; serum IgA antibodies of patients with untreated CD have been reported to strongly react against human brain-blood vessel structures, and this mechanism has been hypothesized to be involved in the abnormal nervous system manifestations frequently described in association with CD. Recent studies have demonstrated that anti-gliadin autoantibodies react with common epitopes on gliadin, calreticulin, enterocytes, and with a nuclear autoantigen expressed in intestinal endothelial cells and in fibroblasts. ${ }^{15-18}$ On the other hand, tTG, recognized as the target antigen of CD-specific autoantibodies, is an extracellular enzyme that is distributed in the cells of all organs. This is a possible link between tTG and cardiac damage. ${ }^{12}$ An interesting finding in this study is the clear demonstration that the risk of autoimmune disorders is significantly more elevated in untreated $\mathrm{CD}$ and that the prevalence of autoimmune disorders in $\mathrm{CD}$ patients is related to the duration of exposure to a gluten-containing diet; compared with healthy subjects, patients with early diagnosis of CD do not show an increased prevalence of autoimmune disorders. This observation suggests the need for early diagnosis, prompt implementation of a gluten-free diet, and strict compliance to gluten withdrawal.

\& Key concepts and clinical implications:

Patients with biopsy-proven myocarditis, especially in the presence of clinical findings of malabsorption, should be screened for gluten sensitivity and celiac disease. In fact, if gluten sensitivity and $\mathrm{CD}$ are associated with autoimmune myocarditis, administration of a gluten-free diet can significantly improve the clinical outcome. These patients do not usually respond to conventional therapy used for heart failure.

- Patients with myocarditis who do not respond to conventional therapy should be screened for neuroimmunology of gluten sensitivity, celiac disease and the associated antibodies.

\section{A2. Gluten sensitivity and its association with osteoporosis}


Osteopenia and osteoporosis are well-recognized complications of CD and constitute a major problem through their association with bone fractures, mainly in the peripheral skeleton. Many factors have been suggested to play a role in the development of osteopenia in patients with $\mathrm{CD}$, including calcium and vitamin $\mathrm{D}$ malabsorption, malnutrition, and menopause. However, bone demineralization has been demonstrated in patients with no evidence of malabsorption. Therefore, the precise Pathophysiological and molecular mechanisms inducing bone disease in gluten-sensitive patients are still only partially known. It is now well established that gluten sensitivity is caused by a Tcell-mediated hypersensitivity with the subsequent release of imbalanced quantities of proinflammatory and anti-inflammatory cytokines. Because bone loss is an effect observed in a tissue distant from the primary site of inflammation (small intestine), it was speculated that the effect could be attributable to systemic immunological factors. Another possibility that has not been explored until very recently is molecular mimicry between target antigens of gluten sensitivity such as tissue transglutaminase and bone structures. ${ }^{19,20}$

Interestingly, tissue transglutaminase, which belongs to a very ubiquitous family of enzymes catalyzing a $\mathrm{Ca}^{2+}$-dependent acyl-transfer reaction in which new $\gamma$-amide bonds are formed, has been shown to be relevant in bone calcification. Hence, it has been demonstrated that native bone tTG has a specific and key role in modulating maturation of bone/cartilage matrix and facilitating its stabilization and finally mineralization. This effect has been attributed to the tTG cross-linking activity, which generates deamidation and negative charges on specific bases, in which calcium and hydroxyapatite will deposit. In a recent study, ${ }^{19}$ the presence of circulating anti-bone antibodies in sera from patients with $\mathrm{CD}$ was investigated by analyzing the immunoreactive profile of these autoantibodies using immunofluorescence staining and Western blot. Furthermore, it was determined that tissue transglutaminase was the main target of these autoantibodies. Finally, the study explored the relationship between anti-bone antibodies and the presence of bone impairment in these patients. ${ }^{19}$

In fact, sera from $51.5 \%$ of gluten sensitive patients had antibodies that recognized antigenic structures in chondrocytes and the extracellular matrix along mature cartilage, bone interface, and perichordium of bone. Among controls, only two osteoporotic patients showed very low titers of anti-bone autoantibodies. The immunostaining was localized in areas where an active mineralization process occurred and was similar to the distribution of the native bone tissue transglutaminase. The frequency of patients with positive baseline titers of anti-bone antibodies diminished significantly after treatment with a gluten-free diet. This study provided original evidence that patients with celiac disease have IgA-type circulating autoantibodies against intraand extracellular structures of tibia. Further, it was suggested that these antibodies recognize bone tissue transglutaminase as the autoantigen, and based on the localization of the immunoreactivity, it was speculated that they might have an active role in the pathophysiology of celiac disease-associated bone complications, including osteopenia and osteoporosis. ${ }^{19}$

\& Key concepts and clinical implications: 
- Original evidence showed that most patients with gluten sensitivity have circulating autoantibodies against bone structures and that bone tTG may be one of the autoantigens involved in sera immunoreactivity.

- High titers of antibodies correlate with the most severe bone impairment and are significantly reduced after treatment with a gluten-free diet.

- Localization of anti-bone antibodies with areas of active mineralization suggests a possible role for these antibodies in bone disorders.

- Patients with osteoporosis and osteopenia should be tested for neuroimmunology of gluten sensitivity, celiac disease and the associated autoantibodies.

\section{A3. Association between gluten sensitivity and neuroautoimmunity}

During the past two decades, gluten sensitivity and CD has been recognized as a multisystem autoimmune disorder. A growing body of distinct neurologic conditions such cerebellar ataxia, epilepsy, myoclonic ataxia, chronic neuropathies, and dementia have been reported. However, recent studies suggest that the variability of neurologic disorders that occur in gluten sensitivity is broader than previously reported and includes "softer" and more commn neurologic disorders, such as chronic headache, developmental delay (autism), hypotonia, and learning disorders or ADHD. ${ }^{2,21}$

In relation to these diseases, a question has been raised: Could neural-cell degeneration, resulting in antibody formation as an epiphenomenona, be the trigger to an immune response to gluten at the lamina propria of the gut? After all, the reverse has been shown to be true: anti-gliadin antibodies cross-react with epitopes on Purkinje cells. If such a contention is true, the explanation might well come from the transglutaminase story discussed earlier in relation to gliadin and bone antibody. ${ }^{19,20}$

However, the evidence for gluten ataxia as a disease entity is now overwhelming. The disease is characterized by ataxia, the presence of anti-gliadin antibodies, the HLA haplotype (DQ2, DQ8) asociated with gluten sensitivity, the presence of anti-Purkinje cell antibodies, the presence of high levels of the interferon- $\gamma$-inducible chemokine CXCL10 and often oligoclonal bands in the cerebrospinal fluid (CSF) and the presence of inflamamtory pathology of the cerebellum at postmortem. ${ }^{2,21-25}$ Perhaps even more compelling is the evidence of a clinical response in the form of improvement of ataxia after a gluten-free diet, even in the absence of an enteropathy. This was demonstrated in the largest control study ever to be published indicating the relationship between gluten, cerebellar antibody and the presence of ataxia. ${ }^{26-29}$

\section{What is the pathogenesis of neurological dysfunction in gluten sensitivity?}

Experimental evidence suggests that there is antibody cross-reactivity between antigenic epitopes on Purkinje cells and gluten peptides. Thus, serum from patients with $\mathrm{CD}$ but no neurological symptoms demonstrate cross-reactivity with epitopes on Purkinje cells. The reactivity can be abolished after adsorption of the anti-gliadin antibodies with 
crude gliadin. In the sera of patients with gluten ataxia, however, there is evidence of additional antibodies targeting Purkinje-cell epitopes. ${ }^{27}$

To investigate the epitope responsible for cross-reaction between gliadin peptides and cerebellar peptides, in our study we assessed the reactivity of sera from 50 autism patients and 50 healthy controls to specific peptides from gliadin and the cerebellum. ${ }^{30} \mathrm{~A}$ significant percentage of autism patients showed elevations in antibodies against gliadin and cerebellar peptides simultaneously. For examining cross-reaction between dietary proteins and cerebellar antigens, antibodies were prepared in rabbits, and binding of rabbit anti-gliadin, anti-cerebellar peptides, anti-MBP, anti-milk, anti-egg, anti-soy and anti-corn to either gliadin- or cerebellar-antigen-coated wells was measured. In comparison to anti-gliadin peptide binding to gliadin peptide at $100 \%$, the reaction of anti-cerebellar peptide to gliadin peptide was $22 \%$, whereas the binding of anti-myelin basic protein (MBP), anti-milk, anti-egg and anti-soy to gliadin was less than $10 \%$.

Further examination of rabbit anti-gliadin 8 amino acid peptide (EQVPLVQQ) and anti-cerebellar 8 amino acid peptide (EDVPLLED) with human serum albumin and an unrelated peptide showed no binding, but the reaction of these antibodies with both the cerebellar and gliadin peptides was greater than $60 \%$. This cross-reaction was further confirmed by DOT-immunoblot and inhibition studies. We concluded that a subgroup of patients with autism produce antibodies against Purkinje cells and gliadin peptides, which may be responsible for some of the neurological symptoms in autism. ${ }^{30}$

\section{A4. Binding of anti-gliadin antibody to neuronal synapsin}

Synapsin is a neuronal phosphoprotein involved in the regulation of neurotransmitter release. Celiac disease is also characterized by systemic manifestations that contribute to a complex clinical presentation. Neurologic deficits, including axonal neuropathy and cerebellar ataxia, are among the most common extraintestinal symptoms associated with celiac disease which were discussed earlier. Based on these neurological manifestations of gluten sensitivity, a different study looked into the cross-reactivity of anti-gliadin humoral immune response with neural tissue. ${ }^{31}$ It was shown that both human and animal anti-gliadin antibodies can cross-react with synapsin I, a cytosolic phosphoprotein found in most neurons of the central and peripheral nervous systems. The anti-gliadin antibodies bound to both isomers of synapsin I, a and b, which have very similar amino acid sequences. ${ }^{31}$

In the human serum samples, antibody to synapsin I was detected in several patients with gluten sensitivity, while control specimens without anti-gliadin antibody did not exhibit significant anti-synapsin antibody reactivity. The patient data also clearly demonstrated that anti-gliadin antibody levels do not necessarily correlate with antisynapsin antibody reactivity and that only certain subsets of anti-gliadin antibodies crossreact with synapsin I. Because of the large number and heterogeneous nature of gliadins, as well as the high diversity of wheat phenotypes, the anti-gliadin immune response is likely to involve a sizeable repertoire of antigenic determinants. Therefore, varying degrees of cross-reactivity to synapsin I can be expected in different patients with gluten sensitivity. ${ }^{32}$ Such differences in the anti-gliadin antibody cross-reactivity in different patients may reveal clues about the potential pathogenic role of the antibody and its association with specific extra-intestinal complications. 
Although pathogenic antibodies typically target antigens in the extracellular matrix or on the cell surface, there is evidence that antibodies to intracellular antigens can also cause disease. For example, antibodies to glutamic acid decarboxylase, which catalyzes the production of the neurotransmitter $\gamma$-aminobutyric acid, have been shown to selectively suppress $\gamma$-aminobutyric acid-mediated synaptic transmission. As synapsin I is associated with synaptic vesicles, it might be similarly targeted by antibodies taken up from the extracellular compartment. ${ }^{33-36}$ Therefore, it is conceivable that, in some patients with gluten sensitivity, the anti-gliadin antibody response would affect synapsin I activity, thus interfering with neurotransmitter release and resulting in neurologic dysfunction. ${ }^{35-37}$

Immune cross-reactivity may also lead to tissue damage through $\mathrm{T}$ cell-mediated mechanisms. Among the celiac patients in this recent study, anti-synapsin antibodies were present in subjects with neurologic disease, as well as those without. ${ }^{31}$ This implies that, like other autoimmune disorders, antibody reactivity is only one piece of the puzzle in the pathogenic mechanism of the neurologic complications of celiac disease. Therefore, the potential pathogenic role of antisynapsin immune cross-reactivity in the neuropathy or CNS manifestations is likely to depend on a number of additional factors, including the type and fine specificity of the immune response, local integrity of the blood-nerve or blood-brain barrier, and presence of proinflammatory factors.

\& Key concepts and clinical implications:

Antibody against gliadin from patients with gluten sensitivity reacts with myelin basic protein, neurofilaments, and cerebellar peptides.

Antibody against gliadin from patients with gluten sensitivity reacts with neural protein called synapsin I.

- Presence of anti-synapsin antibody in some patients with gluten sensitivity can affect synapsin I activity, interfere with neurotransmitter release, and result in neurologic dysfunction.

Patients with abnormal levels of neurotransmitter, and signs and symptoms of neurological disorders should be tested for neuroimmunology of gluten sensitivity, celiac disease, and associated autoantibodies, including neural cell antibodies.

\section{A5. Gluten sensitivity and antibodies against glutamic acid decarboxylase, an enzyme responsible for the production of GABA}

In addition to tissue transglutaminase, glutamic acid decarboxylase (GAD) is another target enzyme involved in gluten sensitivity and neuroauotimmune disorders. GAD is the enzyme responsible for the production of $\gamma$-aminobutyric acid (GABA), the most abundant inhibitory neurotransmitter in the central nervous system. ${ }^{38,39}$ Antibodies against GAD have been described in stiff person syndrome, insulin-dependent diabetes mellitus (IDDM), and autoimmune polyendocrine syndromes, as well as in some immune 
mediated ataxias. These antibodies are present in at least $60 \%$ of both patients with gluten ataxia and patients with $\mathrm{CD}$ and no neurological manifestations. Furthermore, the levels and positivity of these anti-GAD antibodies can be significantly reduced by the introduction of a gluten-free diet in both of these patient groups. Of the patients with neurological manifestations, who also have an enteropathy, the prevalence of these antibodies is $96 \%$. These observations imply that the presence of these antibodies in the context of the enteropathy might predispose individuals to the development of neurological disease. ${ }^{40}$ However, this cannot explain the entire story within the whole spectrum of gluten sensitivity because the antibodies are still present in some patients with gluten-related neurological dysfunction and no enteropathy. Patients with disease processes characterized by the presence of anti-GAD antibodies also appear to have a higher prevalence of CD (e.g. patients with IDDM), stiff person syndrome and polyendocrine syndrome type II, where CD can be part of the syndrome). The prevalence of GAD within the nervous system correlated with the clinical presentation of ataxia and/ or peripheral neuropathy being the commonest neurological manifestations of gluten sensitivity. The presence of GAD in the enteric plexus could hold the key to the generation of anti-GAD antibodies in patients with CD. ${ }^{38-40}$

of Key concepts and clinical implications:

- In addition to gliadin and transglutaminase antibodies, patients with neurological symptomatologies should be tested for glutamic acid decarboxylase (GAD), myelin basic protein, neurofilaments, and cerebellar antibodies.

\section{A6. Gluten sensitivity and autoimmune endocrine disorders}

Polyglandular autoimmunity. Autoimmune polyglandular syndrome is a rare endocrine disorder comprising a combination of at least two of the following autoimmune endocrine disorders: Addison's disease, autoimmune thyroid diseases, hypoparathyroidism, type I diabetes, or primary gonadal failure. ${ }^{41-44}$ In addition, nonendocrine diseases that have been described in autoimmune polyglandular syndrome include mucocutaneous candidiasis, vitiligo, alopecia, pernicious anemia, autoimmune hepatitis, primary biliary cirrhosis, and intestinal malabsorption. There have been several studies on a link between autoimune polyglandular syndrome (including type I diabetes), thyroiditis, and gluten sensitivity. ${ }^{16,41-49}$ Therefore, gluten sensitivity may coexist with several extraintestinal diseases.

Many studies indicate an association between type I diabetes and celiac disease, as is summarized in reference \#50. This prevalence of CD in type I diabetes patients is 10 to 30 times that of the normal population. Since many type I diabetes have asymptomatic or silent CD or gluten sensitivity without enteropathy, this association is recognized in such a highly susceptible population only after administration of a gluten-free diet and an improvement in a diabetic condition; for example, decreased frequency of insulin reactions. The disappearance of diabetic instability after the introduction of a gluten-free diet in such patients emphasizes the importance of the early recognition and identification of CD. ${ }^{50-51}$

Thyroid autoimmunity is another example of an autoimmune disease that is associated with celiac disease. It is common and is due to an apparent immune reaction 
directed against self antigens of the thyroid. Three thyroid sieases are considered to have autoimmune etiology: Hashimoto's thyroiditis, idiopathic myxedema, and Grave's disease. The antigens against which the autoimmune reactions are directed to produce thyroid autoimmune disease include thyroglobulin ( $\mathrm{Tg}$ ), thyroid peroxidase (TPO), and the TSH receptor. ${ }^{52-55}$ It seems that these autoantibodies cause direct thyroid dysfunction, as in Grave's disease caused by antibodies to the TSH receptor, or a destructive process, as in Hashimoto's thyroiditis and idiopathic myxedema.

$\mathrm{CD}$ and autoimmune thyroid disorders share a common genetic predisposition, namely, the DQ2 allele. This common predisposing genetic background would explain the higher incidence of thyroid autoimmune disorders in CD than in the general population. For example, in one of many studies, the investigator found that the overall prevalence of autoimmune thyroid diseases was significantly higher in celiac patients than in controls ( $21 \%$ versus $11 \%)$. The prevalence of both hypo- and hyperthyroidism was not different from that of controls, while the prevalence of autoimmune thyroid disease with euthyroidism was $13 \%$ in patients and $4.7 \%$ in controls. ${ }^{47}$ In a different study, investigators performed thyroid antibody tests and thyroid echography in 47 patients with $\mathrm{CD}$ and 91 healthy controls and found that $\mathrm{CD}$ patients had a three to fourfold increase in the incidence of thyroid autoimmunity ${ }^{45}$ Moreover, several studies have reported an association of idiopathic hypoparathyroidism with CD. CD has been reported to be associated with both hypo- and hyper- (both primary and secondary) thyroidism. CD has been associated with significant bone loss and hypocalcemia. Most $\mathrm{Cd}$ patients with bone loss and hypocalcemia have secondary hypoparathyroidism. A gluten-free diets in patients with $\mathrm{CD}$ with secondary hypoparathyroidism has been reported to result in normal bone mineral density. Therefore, the presence of hypocalcemia or normocalcemic hypoparathyroidism should prompt an examination for CD. Treatment with a gluten-free diet should result in clinical improvement and restoration of normal calcium levels. ${ }^{56-61}$

It is thus recommended that patients with autoimmune endocrine disorders (including patients with autoimmune thyroiditis, type I diabetes, Addison's disease) and patients with idiopathic hypo- and hyperparathyroidism be routinely investigated for gluten sensitivity, with or without enteropathy. If simultaneously antibodies against glutamic acid decarboxylase, transglutaminase, thyroglobulin, thyroid peroxidase, heat shock protein and gliadin, gliadin peptide and gluteomorphins are detected, then the patient should be monitored closely after the instution of a gluten-free diet. All these antibodies could be measured by a highly specialized laboratory under neuroimunology of gluten sensitivity and celiac disease.

of Key concepts and clinical implications:

The incidence of $\mathrm{CD}$ in various autoimmune disorders is increased 10- to 30-fold in comparison to the general population. The identification of such cases with CD is important since it may help in the control of type I diabetes or endocrine functions in general, as well as in the prevention of long-term complications of $\mathrm{CD}$, such as lymphoma. It is believed that $\mathrm{CD}$ may predispose an individual to other autoimmune disorders such as type I diabetes, autoimmune thyroid, and other endocrine diseases, and that gluten may be a possible trigger. 
- The performance of Neuroimmunology of Gluten Sensitivity and celiac disease may be considered for patients with $\mathrm{CD}$ who may have different autoimmune conditions or for patients with autoimmune endocrine disorders who may suffer from gluten sensitivity.

- This neuroimmunology panel not only includes antibodies against gliadin, gluteomorphin, parietal cell and transglutaminase, which limit their findings only to the gut, but also emphasized the importance of its extraintestinal consequences. For this reason, antibodies against glutamic acid decarboxylase, thyroglobulin, thyroid peroxidase, heat shock protein, myelin basic protein, neurofilaments, cerebellar and many other antigens are measured in order to detect neuroimmune disorders in patients with gluten sensitivity.

- This novel laboratory approach to gluten sensitivity and autoimmunity will enable clinicians to detect markers of disease years before the onset of autoimmune disease.

- Early identification of CD patients in a highly susceptible population may result in the treatment of subclinical CD and improved control of associated disorders.

\section{References}

1. Marks J., et al. Small bowel changes in dermatitis herpetiformis. Lancet, 1966; 2:1280-1282.

2. Cooke W.T., and Thomas-Smith W. Neurological disorders associated with adult celiac disease. Brain, 1966; 89:683-722.

3. Hvatut M., et al. The gut-joint axis: cross-reactove food antibodies in rheumatoid arthritis. Gut, 2006; 55:1240-1247.

4. Pattison D.J., et al. Dietary risk factors for the development of inflammatory polyarthritis: evidence for a role of high level of red meat consumption. Arthritis Rheum, 2004; 50:3804-3812.

5. van de Laar M.A., and van der Korst J.K. Food intolerance in rheumatoid arthritis. I. A double blind, controlled trial of the clinical effects of elimination of milk allergens and azo dyes. Ann Rheum Dis, 1992; 51:298-302.

6. Kjeldsen-Kragh J., et al. Antibodies against dietary antigens in rheumatoid arthritis patients treated with fasting and a one-year vegetarian diet. Clin Exp Rheumatol, $1995 ; 13: 167-172$.

7. Koot V.C., et al. Elevated level of IgA gliadin antibodies in patients with rheumatoid arthritis. Clin Exp Rheumatol, 1989; 7:623-626.

8. O'Farelly C., et al. Association between villous atrophy in rheumatoid arthritis and a rheumatoid factor and gliadin-specific IgG. Lancet, 1988; ii:819-822.

9. Bjarnason I., and Peters T.J. Influence of anti-rheumatic drugs on gut permeability and the gut associated lymphoid tissue. Baillieres Clin Rheumatol, 1996; 10:165-176. 
10. Sökjer M., et al. Selective increase of IgA rheumatoid factor in patients with gluten sensitivity. Acta Derm Venereol, 1995; 75:130-132.

11. Quan C.P., et al. Natural polyreactive secretory immunoglobulin A autoantibodies as a possible barrier to infection in humans. Infect Immun, 1997: 65:3997-4004.

12. Frustaci A., et al. Celiac disease associated with autoimmune myocarditis. Circulation, 2002; 105:2611-1618.

13. Curione M., et al. Prevalence of coeliac disease in idiopathic dilated cardiomyopathy. Lancet, 1999; 354:222-223.

14. Chimenti C., et al. Celiac disease in idiopathic dilated cardiomyopathy. Ital Heart J, 2001; 2:658-659.

15. Collin P., et al. High frequency of coeliac disease in adult patients with type-1 diabetes. Scand J Gastroentero/, 1989; 24: 81-84.

16. Counsell C.E., et al. Coeliac disease and autoimmune thyroid disease. Gut, 1994; $35: 844-846$.

17. Natter S., et al. IgA cross-reactivity between a nuclear autoantigen and wheat proteins suggests molecular mimicry as a possible pathomechanism in celiac disease. Eur J Immunol, 2001; 31:918-928.

18. Pedeira S., et al. Clinical significance of anti-smooth muscle antibody (SMA) fluorescence in patients with celiac disease. Gastroenterol, 2000; 118:A363.

19. Sugal E. Bone-specific antibodies in sera from patients with celiac disease: characterization and implications in osteoporosis. J Clin Immunol, 2002; 22:353-362.

20 . Vazquez H., et al. Risk of fractures in celiac disease patients: A cross-sectional, casecontrol study. Am J Gastroentero1, 2000; 95:183-189.

21. Pratesi R, et al. Serum IgA antibodies from patients with coeliac disease react strongly with human brain blood-vessel structures. Scand J Gastroenterol, 1998; 33:817-821.

22. Volta U., et al.Clinical findings and anti-neuronal anlibodies in celiac disease with neurological disorders. Scand J Gastroenterol, 2002; 137: 1276-1281.

23. Hadjivassiliou M., et al. Gluten ataxia in perspective: epidemiology, genetic susceptibility and clinical characteristics. Brain, 2003; 126:685-691.

24. Hadjivassiliou M. et al. (1998) Clinical, radiological, neurophysiological and neuropathological characteristics of gluten ataxia. Lancet, 1998; 352:1582-1585. 
25. Abele, M. et al. Prevalence of antigliadin antibodies in ataxia patients. Neurology, 2003; 60:1674-1675.

26. Bushara, K. et al. Antigliadin antibodies in Huntington's disease. Neurology, 2004; 62:132-133.

27. Hadjivassiliou, $M$, et al. The humoral response in the pathogenesis of gluten ataxia. Neurology, 2002; 58:1221-1226.

28. Hadjivassiliou, M. et al. Dietary treatment of gluten ataxia. $J$ Neurol Neurosurg Psychiatry, 2003; 74:1221-1224.

29. Pellecchia, M.T. et al. Cerebellar ataxia associated with subclinical celiac disease responding to gluten-free diet. Neurology, 1999; 53:1606-1607.

30. Vojdani A., et al. Immune response to dietary proteins, gliadin and cerebellar peptides in children with autism. Nutritional Neurosciences, 2004; 7(3):151-161.

31. Alaedini, A., et al. immune cross-reactivity in celiac disease: anti-gliadin antibodies bind to neuronal synapsin I. J Immunol, 2007; 178:6590-6595.

32. Green P.H., et al. Mechanisms underlying celiac disease and its neurologic manifestations. Cell Mol Lifte Sci, 2005; 62:191-199.

33. Hilfiker, S., et al. Structural domains involved in the regulation of transmitter release by synapsins. J Neurosci, 2005; 25:2658-2669.

34. Li, L., et al. Impairment of synaptic vessivle clustering and of synaptic transmission, and increased seizure propensity, in synapsin I-deficient mice. Proc Natl Acad Sci USA, 1995; 92:9235-9239.

35. Sommer, C., et al. Paraneoplastic stiff-person syndrome: passive transfer to rats by means of IgG antibodies to amphiphysm. Lancet, 2005; 365: 1406-1411.

36. Takenoshita, H.M, et al. Presynaptic inhibition of cerebellar GABAergic transmission by glutamate decarboxyJase autoantibodies in progressive cerebellar ataxia. J Neural Neurosurg PsychIatry, 2001; 70:386-389.

37. Zelnick N., et al. Range of neurologic disorders in patients with celiac disease. Pediatr, 2004; 113:1672-1676.

38. Hadjivassiliou, M. et al. Glutamic acid decarboxylase as a target antigen in gluten sensitivity: the link to neurological manifestations? Proceedings of the 11th International Symposium on Coeliac Disease, Belfast, 2004.

39. Ho1mes, G.K.T. (2001) Coeliac disease and Type 1 diabetes mellitus - the case for screening. Diabet. Med. 18, 169-177. 
40. Hadjivassiliou M., et al. The immunology of gluten sensitivity beyond the gut. Trends Immunol, 2004; 25:578-582.

41. Cronin C.C., and Shanahan F. Insulin-dependent diabetes mellitus and coeliac disease. Lancet, 1997; 349:1096-1097.

42. Kapuscinska A., et al. Disease specificity and dynamics of changes in IgA class antiendomysial antibodies in celiac disease. J Pediatr Gastroenterol Nutr, 1987; 6:529-534.

43. Kordonouri O., et al. Autoantibodies to tissue transglutaminase are sensitive serological parameters for detecting silent coeliac disease in patients with type I diabetes mellitus. Diabetes Med, 2000; 17:441-444.

44. Sategna-Guidetti C., et al. Celiac disease and insulin-dependent diabetes mellitus. Screening in an adult population. Dig Dis Sci, 1994; 39:1633-1637.

45. Lorini R., et al. Celiac disease and type I (insulin-dependent) diabetes mellitus in childhood: follow-up study. J Diabetes Complications, 1996; 10:154-159.

46. Velluzzi F., et al. Thyroid and celiac disease: clinical, serologic and echographic study. Am J Gastroenterol, 1998; 93:976-979.

47. Sategna-Guidetti C., et al. Autoimmune thyroid disease and coeliac disease. Eur $J$ Gastroenterol Hepatol, 1998; 10:927-931.

48. Stagi S., et al. Thyroid function, autoimmune thyroiditis and coeliac disease in juvenile idiopathic arthritis. Rheumatology, 2005; 44:517-520.

49. Kumar V., et al. Celiac disease associated autoimmune endiocrinopathies. Clin Diag Lab Immunol, 2001; 8:678-685.

50 . Funda D.P., et al. Gluten-free diet prevents diabetes in NOD mice. Diabetes Metab Res Rev, 1999; 15:323-327.

51. Westman E., et al. Children with coeliac disease and insulin-dependent diabetes mellitus-growth, diabetes control dietary intake. J Pediatr Endocrinol Metab, 1999; 12:433-442.

52. Venture A., et al. Gluten-dependent diabetes-related and thyroid-related antibodies in patients with celiac disease. J Pediatr, 2000; 137:263-265.

53. Valdimarsson T., et al. Three years' follow-up of bone density in adult coeliac disease: significance of hyperparathyroidism. Scand $J$ Gasroenterol, 2000; 35:274-280. 
54. Valentino R., et al. Unusual association of thyroiditis, Addison's disease, ovarian failure and celiac disease in a young woman. $J$ Endocrinol Investig, 1999; 22:390-394.

55. Valentino R., et al. Prevalence of coeliac disease in patients with thyroid autoimmunity. Horm Res, 1999; 51:124-127.

56. Selby P., Bone loss in celiac disease is related to secondary hyperparathyroidism. $J$ Bone Mineral Res, 1999; 14:652-657.

57. Nilsen E., et al. Gluten-specific HLA-DQ restricted T cells from coeliac mucosa produce cytokines with Th1 or Th0 profile dominated by interferon gamma. Gut, $1995 ; 37: 766-776$.

58. Iafusco D., et al. Effect on gluten-free diet on the metabolic control of type I diabetes in patients with diabetes and celiac disease. Diabetes Care, 2000; 23:712-713.

59. Takeda R., et al. Schmidt's syndrome: autoimmune polyglandular disease of the adrenal and thyroid glands. Isr Med Assoc J, 1999; 285-286.

60. Talal A., et al. Celiac disease in an adult population with insulin-dependent diabetes mellitus - use of endomysial antibody testing. Am J Gastroenterol, 1997: 92:1280-1284.

61. Toscano V., et al. Importance of gluten in the induction of endocrine autoantibodies and organ dysfunction in adolescent celiac patients. Am J Gastroenterol, 2000: 95:1742-1748. 